\title{
Infrared and Nuclear Magnetic Resonance Spectrometry of Caffeine in Roasted Coffee Beans after Separation by Preparative Supercritical Fluid Chromatography
}

\author{
Patrick Elisabeth*, Masanori Yoshioka*, Yoshio YaMauchi** and Muneo Saito**† \\ *Department of Analytical Chemistry, Faculty of Pharmaceutical Sciences, Setsunan University, \\ Nagaotogecho, Hirakata, Osaka 573-01, Japan \\ **JASCO (Japan Spectroscopic Co., Ltd.), Ishikawacho, Hachioji, Tokyo 192, Japan
}

\begin{abstract}
The isolation of caffeine from coffee powder was performed using supercritical fluid extraction (SFE) and preparative supercritical fluid chromatography (SFC). Two grams of roasted coffee bean powder gave $0.49 \mathrm{~g}$ of extract using supercritical carbon dioxide $\left(40^{\circ} \mathrm{C}, 200 \mathrm{~kg} / \mathrm{cm}^{2}\right)$ as an extractant. This extract was mixed with $0.6 \mathrm{ml}$ of methanol and after decantation, $0.2 \mathrm{ml}$ of the supernatant was subjected to preparative SFC. Fractionation was carried out with referring to the retention time of the standard caffeine. After evaporation of ethanol added as a modifier from the collected fraction, a 7-8 mg of needle-like crystals was obtained. This crystal was identified to be caffeine by FTIR and by FT-NMR, respectively.
\end{abstract}

Keywords Caffeine, supercritical fluid extraction, supercritical fluid chromatography, Fourier transform infrared spectrometry, Fourier transform nuclear magnetic resonance spectrometry

Coffee is certainly one of the most frequently consumed beverages not only in North America but all over the world. ${ }^{1}$ It is well known that coffee contains caffeine (1,3,7-trimethylxanthine), which is considered to be its most physiologically active component. ${ }^{2}$ In addition, caffeine is present in tea, mate leaves, cocoa, and cola nuts. ${ }^{3}$

Supercritical fluid extraction (SFE), which uses a supercritical fluid as an extraction medium, has long been used to remove caffeine from green coffee beans in industrial decaffeination processes. ${ }^{4}$

Sugiyama et al. demonstrated directly coupled SFE/ SFC (supercritical fluid chromatography) analysis of roasted coffee beans, showing that various compounds could be extracted, including caffeine. ${ }^{5}$ They also investigated such optimum conditions as the pressure, temperature, water content and extraction time for caffeine extraction by off-line SFE/HPLC (high performance liquid chromatography). ${ }^{5}$

Since these studies were performed in view of analytical separation, they did not mean to recover sufficient amounts of extracts for further molecular spectroscopic analyses, such as infrared (IR) and nuclear magnetic resonance (NMR) spectrometry for identification.

We investigated how to perform SFE and SFC in larger scale so that it would be possible to recover caffeine fraction for further identification analyses. The amount of obtained caffeine was enough to identify by

$\uparrow$ To whom correspondence should be addressed.
Fourier transform infrared spectrometry (FTIR) and by NMR spectrometry.

\section{Experimental}

\section{Reagents and a column}

Anhydrous caffeine $\left(\mathrm{C}_{8} \mathrm{H}_{10} \mathrm{~N}_{4} \mathrm{O}_{2}\right)$ was obtained from Wako Pure Chemicals (Osaka, Japan). Coffee powder was UCC original blend coffee (medium fine grind) obtained from UESHIMA COFFEE (Kobe, Japan). Carbon dioxide was obtained from Toyoko Kagaku (Kawasaki, Japan). Ethanol and methanol were also from WAKO Pure Chemicals. Water used was purified from tap water using a Millipore Milli-R/ $Q$ (Massachusetts, USA) water purification system. SFC was performed using a $5 \mu \mathrm{m}$ silica gel column $(10 \mathrm{~mm}$ i.d. $\times 250 \mathrm{~mm}$ length) (JASCO, Tokyo, Japan). The $\mathrm{KBr}$ crystal used for IR measurements was from JASCO (Tokyo, Japan).

\section{Apparatus}

SFE and SFC were carried out using a JASCO Model SUPER-200 SFE/SFC System-2, comprising a carbon dioxide and modifier delivery section; an extraction/ chromatography section equipped with a Rheodyne injector Model 7125 (Cotati, CA, USA) with a $200 \mu \mathrm{l}$ sampling loop; and a JASCO Model 880-81 backpressure regulator.

A UV/VIS multiwavelength detector (JASCO Model 
MULTI-330) was used for monitoring extraction and chromatographic profiles. DP-L320/98 data processing software was used for data analysis on an NEC PC9801VM personal computer (NEC, Tokyo, Japan).

Infrared spectral measurements were performed using a microsampling FTIR spectrometer (JASCO Model Micro FTIR-100) with a workstation (JASCO Model WS/IR-8000). NMR spectrometric measurements were performed by using a JEOL EX 270 FT NMR (Akishima, Tokyo, Japan).

\section{Procedures}

Coffee pretreatment. Two hundred microliters of water were added to $2 \mathrm{~g}$ of coffee powder, according to Sugiyama et al. ${ }^{5}$, has been vigorously shaken for $15 \mathrm{~min}$, agitated with a vortex for $15 \mathrm{~min}$, and then left for $3 \mathrm{~h}$ before extraction. This process was used to give $c a .10 \%$ water content to the powder.

$S F E$ of coffee. SFE was performed using a $10 \mathrm{ml}$ extraction vessel (JASCO Model EV-2). The coffee extract was collected in a $10 \mathrm{ml}$ glass tube connected to the back-pressure regulator by a spring force. ${ }^{6}$

SFC of coffee extract. The extract obtained in SFE was mixed with $600 \mu \mathrm{l}$ of methanol and vigorously shaken for $5 \mathrm{~min}$, and then agitated with a vortex for $5 \mathrm{~min}$. After decantation, the supernatant was subjected to preparative SFC separation. A standard solution was prepared by dissolving $4 \mathrm{mg}$ of anhydrous caffeine into $1 \mathrm{ml}$ of methanol. This solution was used for identification of the caffeine peak in the chromatogram of the extract by comparing its retention time with a peak possibly of caffeine in the obtained chromatogram. Caffeine fractionation was performed by collecting a portion of the column effluent with reference to the standard chromatogram. Evaporation of modifier solvent from the collected fractions was carried out using a rotary vacuum evaporator in combination with a thermostated water bath.

\section{Results and Discussion}

\section{SFE of coffee}

Figure 1 shows the extraction profile monitored at $275 \mathrm{~nm}$, which is about the maximum absorption wavelength of caffeine. As can be seen, it takes $2 \mathrm{~h}$ to extract $90 \%$ of the compounds which exhibit UV absorption at $275 \mathrm{~nm}$. However, $80 \%$ of the compounds could be extracted within $1 \mathrm{~h}$.

The mass of the coffee powder placed in the extraction vessel was $2.00 \mathrm{~g}$, excluding $10 \%$ added water. This gave $0.49 \mathrm{~g}$ of the extract (oil and xanthine derivatives) and $1.39 \mathrm{~g}$ for the residue, corresponding to a recovery of $80.3 \%$. It is considered that most of the added water was extracted together with other compounds. About $20 \%$ of the extract was gone with carbon dioxide gas to the exhaust line.

Methanol-soluble compounds in the extract were transferred to methanol by the procedure described in

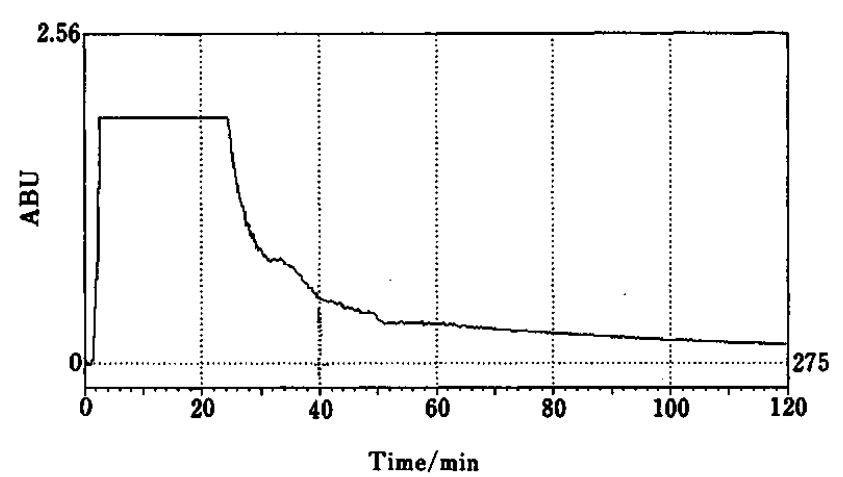

Fig. 1 Extraction profile of roasted coffee beans. Conditions: mobile phase, $\mathrm{CO}_{2}(5 \mathrm{ml} / \mathrm{min})$; temperature, $40^{\circ} \mathrm{C}$; pressure, $200 \mathrm{~kg} / \mathrm{cm}^{2}$; detection, $275 \mathrm{~nm}$.

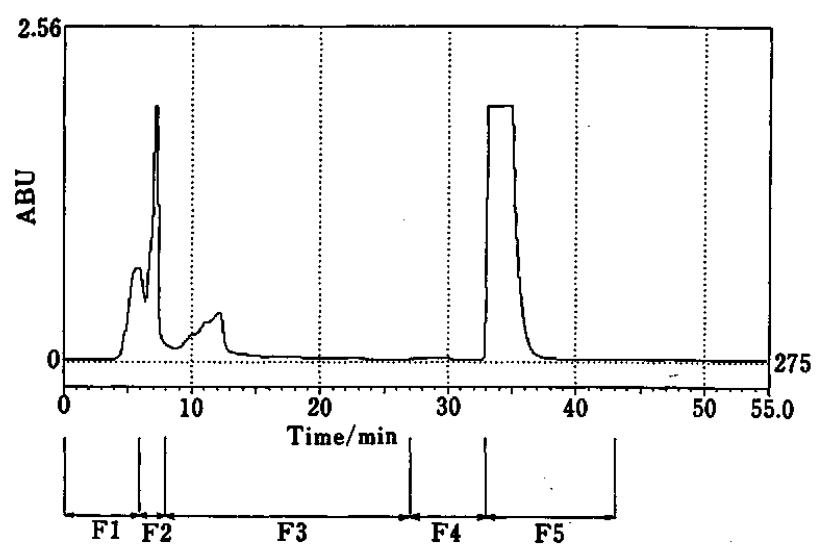

Fig. 2 Chromatogram of coffee extract by SFC. Conditions: mobile phase, $\mathrm{CO}_{2}(5 \mathrm{ml} / \mathrm{min})+$ ethanol $(0.5 \mathrm{ml} / \mathrm{min})$; temperature, $40^{\circ} \mathrm{C}$; pressure, $200 \mathrm{~kg} / \mathrm{cm}^{2}$; sample volume, $200 \mu \mathrm{l}$.

SFC of coffee extract paragraph in Experimental Section.

\section{SFC separation of caffeine from oil in the extract}

Figure 2 shows a chromatogram obtained by injecting $200 \mu \mathrm{l}$ of the supernatant of the extract. The time frames in which the fractions were collected are shown under the time axis of the chromatogram: fraction no. 1 (0-6 min); fraction no. 2 (6-8 min); fraction no. 3 (8$27 \mathrm{~min})$; fraction no. $4(27-33 \mathrm{~min})$ and fraction no. 5 (33-43 min).

Figure 3 shows the chromatogram of the caffeine standard solution obtained under the same conditions. It is shown that the retention time of caffeine is $35.46 \mathrm{~min}$.

Comparison of these chromatograms suggested that fraction no. 5 contained caffeine. Components eluted before $20 \mathrm{~min}$ were less polar compounds than caffeine.

Fraction no. 5 also contained ethanol, which was added as a modifier. After evaporation of ethanol, we obtained pieces of needle-like crystals on the wall of the glass collection vessel. A slightly yellow ring $(0.5 \mathrm{~mm}$ width) attached to the tube suggested the presence of minor impurities. 


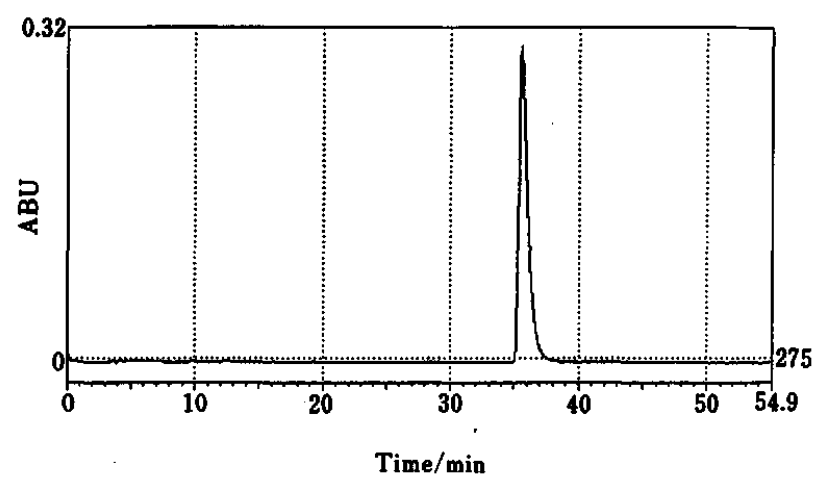

Fig. 3 Chromatogram of standard caffeine. Conditions are same as given in Fig. 2 caption, except that the sample volume was $10 \mu l$.
A sufficient amount (3.5-4 mg) of caffeine was obtained in a single SFC run to perform further identifications by FTIR and FT-NMR analyses.

\section{Identification of caffeine by infrared spectrometry}

Prior to the IR spectrum measurement of the obtained crystal, a measurement of the standard caffeine was performed using the Micro FTIR System by placing the caffeine powder on a $\mathrm{KBr}$ disk (diameter $=3 \mathrm{~mm}$ ). The obtained IR spectrum is shown in Fig. 4A.

We first measured the IR spectrum of the crystal having $45 \mu \mathrm{m}$ diameter by focusing the IR beam on it while being viewed under a microscope. It was found that the crystal scattered too much of the IR beam to obtain the correct IR spectrum. We therefore pressed
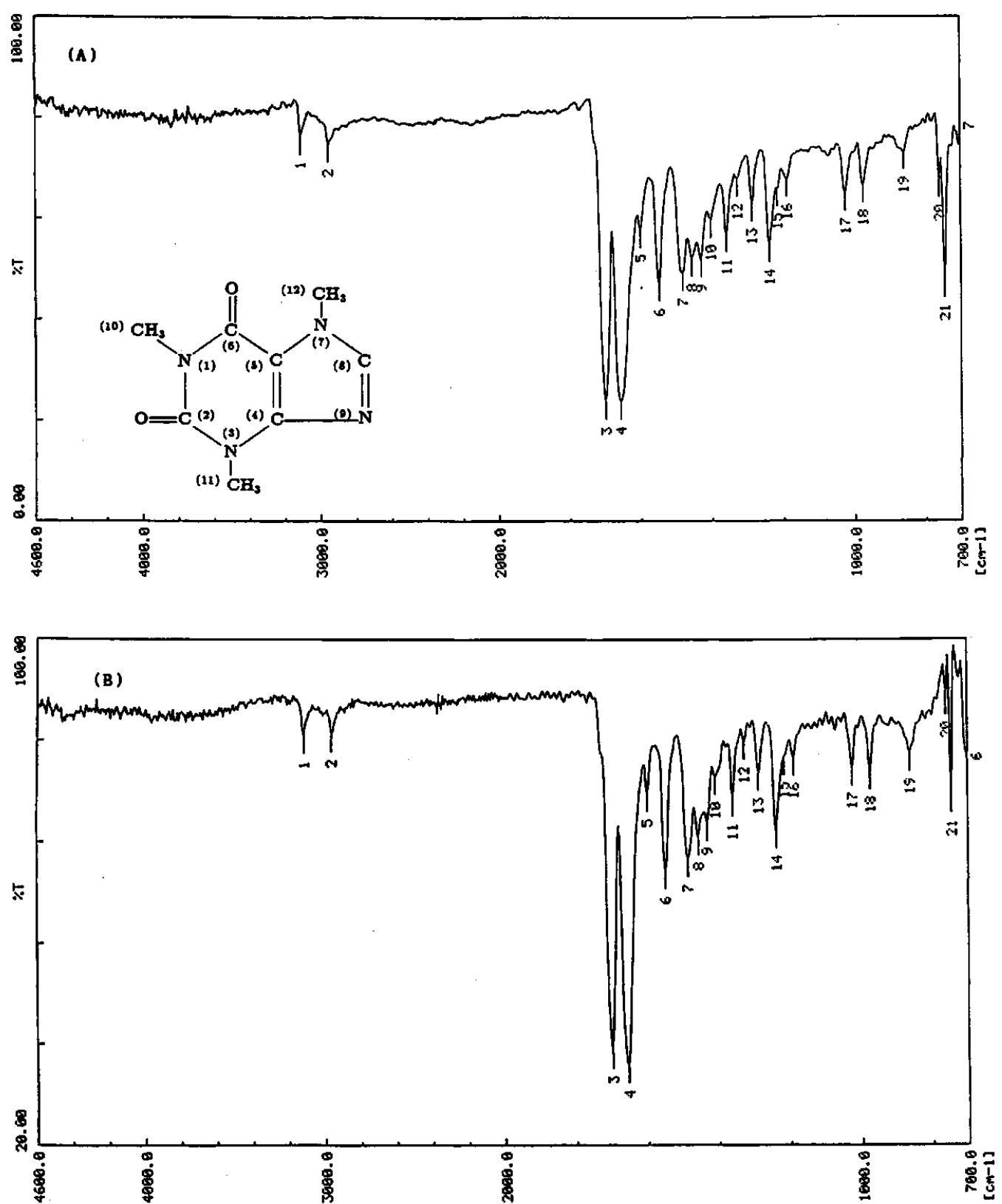

Fig. 4 Micro-FTIR infrared spectra of standard caffeine (A) and isolated caffeine after pressing (B): aperture, $36 \times 45 \mu \mathrm{m}$. 
the crystal with a spatula to crash it into a powder. We could thus obtain a high-quality IR spectrum as shown in Fig. 4B.

The identification of caffeine was performed by comparing the standard and measured IR spectra as shown in Figs. 4A and 4B. The result shows that there is excellent agreement in the wavenumbers of the specific bands of both spectra. The similarity of the shapes of the spectra can be justified by visually comparing the spectra shown in Figs. 4A and 4B. The crystal was thus identified to be caffeine.

\section{Nuclear magnetic resonance measurements}

Figures 5A and 5B show ${ }^{1} \mathrm{H}-\mathrm{NMR}$ spectra of the standard caffeine and isolated caffeine in $\mathrm{CDCl}_{3}$ at $270 \mathrm{MHz}$ (45 degrees pulse with intervals of $2 \mathrm{~s}$ ). The NMR spectrum of the isolated caffeine shown in Fig. 5A has almost the same profile as that of the standard, except for a shift of $1 / 10$ of the ppm. The relative intensities of the peaks are almost the same except for the reference peak and the impurity peak at $5.614 \mathrm{ppm}$ (in the isolated caffeine) of which intensity is three-times higher than that in the standard caffeine spectrum $(5.518 \mathrm{ppm})$. Impurities were found in both spectra. These results suggest that the impurities in the isolated caffeine spectrum originated from the solvent used for NMR measurements, not only from the isolated caffeine. The relationships between the NMR peaks (standard caffeine and a crystal of caffeine) and the functional groups are listed in Table 1.

Figures $6 \mathrm{~A}$ and $6 \mathrm{~B}$ show the ${ }^{13} \mathrm{C}-\mathrm{NMR}$ spectra of the standard caffeine and the isolated caffeine in $\mathrm{CDCl}_{3}$ at $67.5 \mathrm{MHz}$ (45 degrees pulse with intervals of $5 \mathrm{~s}$ ).

The peak shifts in the isolated caffeine show a variation
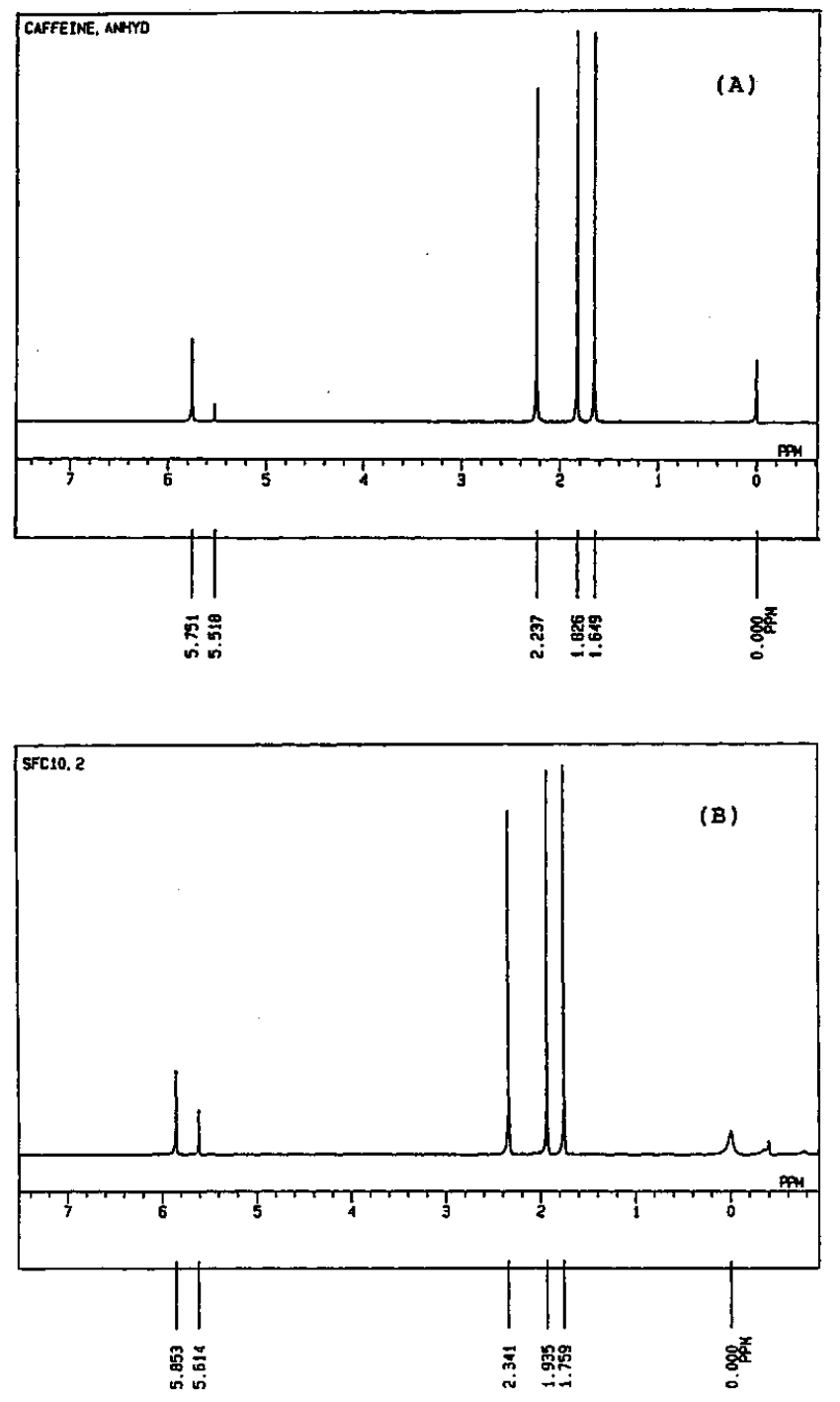

Fig. $5{ }^{1} \mathrm{H}-\mathrm{NMR}$ spectra of standard caffeine (A) and isolated caffeine (B). Conditions: frequency, $270 \mathrm{M} \mathrm{Hz}, 45 \mathrm{deg}$ pulse with intervals of $2 \mathrm{~s}$; Fourier transform data points, 32768; sample tube, $5 \mathrm{~mm}$ i.d.
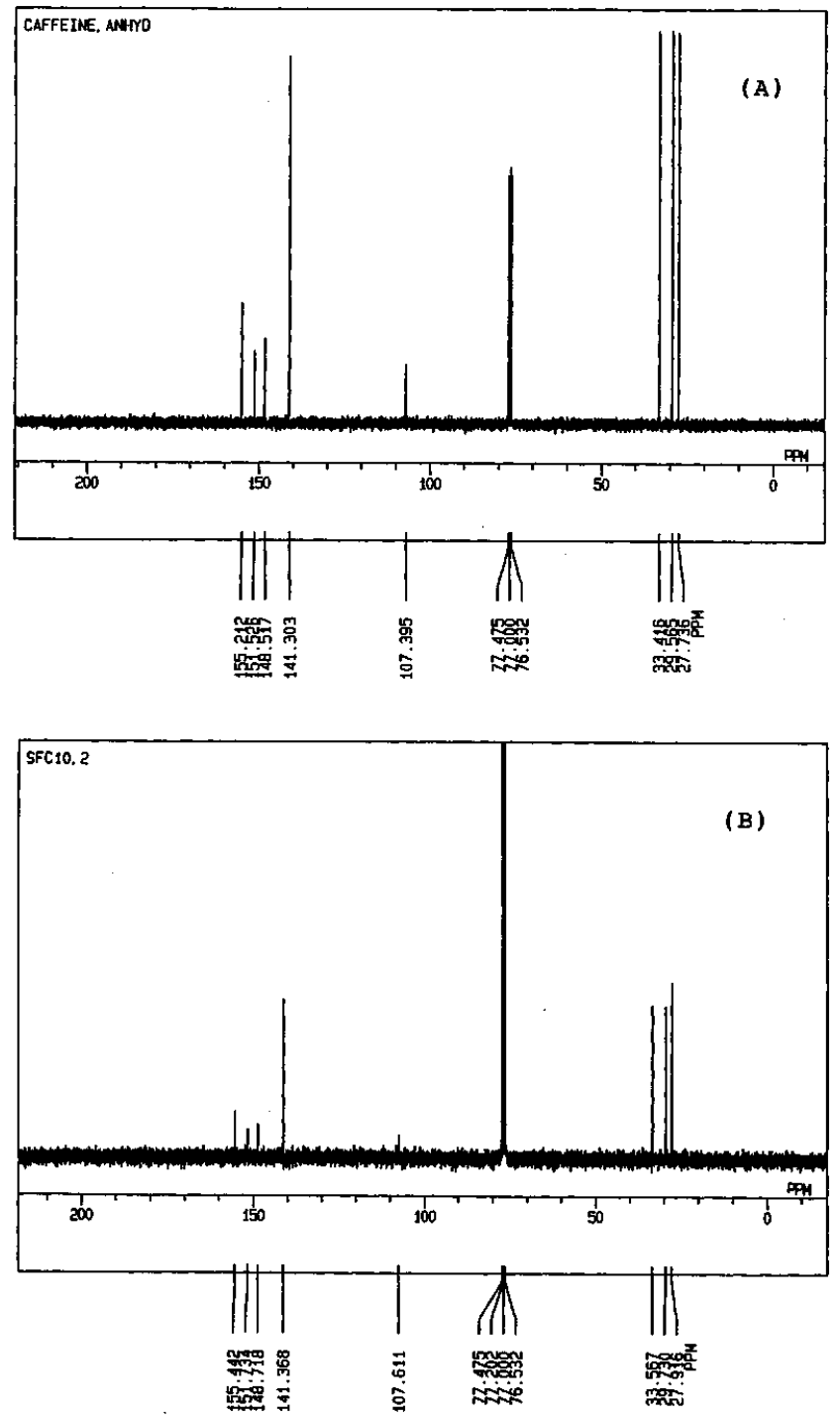

Fig. $6{ }^{13} \mathrm{C}-\mathrm{NMR}$ spectra of standard caffeine (A) and isolated caffeine (B). Conditions: frequency, $67.5 \mathrm{M} \mathrm{Hz}, 45 \mathrm{deg}$ pulse with intervals of $5 \mathrm{~s}$; Fourier transform data points, 65536 . Other conditions were same as given in Fig. 5 caption. 
Table 1 Identification of methyl groups contained in caffeine (standard and extracted) according to chemical shifts in its ${ }^{1} \mathrm{H}-\mathrm{NMR}$ spectra

\begin{tabular}{cccc}
\hline \multicolumn{2}{c}{ Chemical shifts, ppm } & & $\begin{array}{c}\text { Functional groups in } \\
\text { caffeine structure }\end{array}$ \\
\cline { 1 - 2 } Standard & Isolated & & refence TMS \\
\hline 0.000 & 0.000 & & merence \\
1.649 & 1.759 & & methyl group $\mathrm{CH}_{3}^{(11)}$ \\
1.826 & 1.925 & & methyl group $\mathrm{CH}_{3}^{(10)}$ \\
2.237 & 2.341 & methyl group $\mathrm{CH}_{3}^{(12)}$ \\
5.518 & 5.614 & \\
5.751 & 5.853 & $(*)$ \\
\hline
\end{tabular}

(*) impurities. Numbers in parentheses give the place where the functional groups are located in the caffeine structure in Fig. 4A.

of 2/10 of ppm higher than that in the standard caffeine, except for around $141.3 \mathrm{ppm}$ and those due to $\mathrm{CHCl}_{3}$ impurities between 77.475 and $76.532 \mathrm{ppm}$.

Although the peak shift of the $\mathrm{C}(6)$ of the ketone group should have appeared at $167.5 \mathrm{ppm}^{7}$, no such peak shift appeared for this ketone group, because of nuclear Overhauser effect.

The peak intensities were lower in the isolated caffeine than those in the standard caffeine because of the lower sample concentration. An additional peak appeared in the isolated caffeine spectrum at $77.202 \mathrm{ppm}$ with a low intensity compared with the three peaks at $76.532,77.000$ and $77.475 \mathrm{ppm}$, respectively. This peak may have come from the isolated caffeine.

Table 2 summarizes the NMR signal assignments of the functional groups related to the peaks in the caffeine structure.

In this way, the NMR measurements confirmed that the crystal was caffeine.

SFE and SFC allowed the isolation of caffeine from roasted coffee beans as a crystal in less than three hours. Two runs offered about $8 \mathrm{mg}$ of caffeine. This amount was sufficient to allow IR and NMR measurements. In this work, we focused on the isolation and identification of caffeine, however, we also obtained oil fractions which gave the coffee aroma. An analysis of flavor compounds in the SFE extract of coffee is currently under way; the results will be published elsewhere.

The authors thank Ms. F. Kaneuchi, C. Jin and J. Hirata for
Table 2 Identification of carbon atoms contained in caffeine (standard and extracted) according to chemical shifts in its ${ }^{13} \mathrm{C}-\mathrm{NMR}$ spectra

\begin{tabular}{ccc}
\hline \multicolumn{2}{c}{ Chemical shifts, ppm } & \multirow{2}{*}{$\begin{array}{c}\text { Functional groups in } \\
\text { caffeine structure }\end{array}$} \\
\cline { 1 - 2 } Standard & Isolated & methyl group $\mathrm{CH}_{3}^{(12)}$ \\
27.736 & 27.916 & methyl group $\mathrm{CH}_{3}^{(11)}$ \\
29.565 & 29.930 & methyo \\
33.415 & 33.567 & methyl group $\mathrm{CH}_{3}^{(10)}$ \\
76.532 & 76.532 & \\
77.000 & 77.000 & $\left({ }^{*}\right)$ \\
- & 77.202 & \\
77.475 & 77.475 & \\
107.395 & 107.611 & $\left({ }^{* *}\right)$ \\
141.303 & 141.368 & $\mathrm{C}^{(4)}$ \\
148.517 & 148.718 & $\mathrm{C}^{(8)}$ \\
151.526 & 151.734 & $\mathrm{C}^{(2)}$ \\
155.212 & 155.442 & $\mathrm{C}^{(5)}$ \\
\hline
\end{tabular}

$\left(^{*}\right)$ contamination of solvent $\mathrm{CDCl}_{3} ;\left({ }^{* *}\right)$ impurity. Numbers in parentheses give the place where the functional groups or atoms are located in the caffeine structure in Fig. 4A.

instructive discussion concerning infrared spectrum analysis. We are deeply indebted to Prof. K. Jinno of Toyohashi University of Technology and Prof. S. Ogawa of Ochanomizu University for NMR measurements and helpful discussion.

\section{References}

1. R. M. Gilbert in "Nutrition and Behavior", ed. S. A. Miller, p. 145, The Franklin Institute Press, Philadelphia, 1981.

2. L. L. Iverson, Nature [London], 301, 195 (1983).

3. MERCK \& CO., "An Encyclopedia of Chemicals and Drugs", 9th ed., p. 207, Merck \& CO, Inc. Rahway, 1976.

4. K. Zosel, "Extraction with Supercritical Gases", ed. G. M. Schneider, E. Stahl and G. Wilke, p. 1, VCH, Weinheim, 1980.

5. K. Sugiyama, M. Saito, T. Hondo and M. Senda, $J$. Chromatogr., 332, 107 (1985).

6. M. Saito, Y. Yamauchi, H. Kashiwazaki and $M$. Sugawara, Chromatographia, 25, 801 (1988).

7. R. M. Silverstein, G. C. Bassler and T. C. Morrill, "Spectrometric Identification of Organic Compounds", 4th ed., John Wiley \& Sons, New York, 1981.

(Received October 27, 1990) (Accepted March 2, 1991) 